\title{
Bibliometrie
}

Andreas Meier* und Dirk Tunger

\section{Altmetrics - Nutzung, Eindrücke und Erfahrungen am Forschungszentrum Jülich}

https://doi.org/10.1515/iwp-2019-2030

\section{Einleitung}

Alternative Metriken - oder kurz: Altmetrics - beschäftigen die Wissenschaft etwa seit dem Jahr 2010. Ein Konsens darüber, welchen Stellenwert die Metriken besitzen und was man damit überhaupt misst, ist jedoch bis heute nicht greifbar. Nichtsdestotrotz werden Altmetrics nicht nur theoretisch erforscht, sondern auch praktisch verwendet: in erster Linie von Universitäten und wissenschaftlichen Einrichtungen. Sie nutzen Altmetrics vor allem, um die Aufmerksamkeit zu illustrieren, die wissenschaftliche Artikel auf Social Media-Plattformen, in Newsbeiträgen, Policy Documents, Repositorien, Pressemitteilungen, Institutswebseiten oder anderen Netzquellen erhalten.

In diesem Beitrag wird dargestellt, wie Altmetrics im Forschungszentrum Jülich genutzt werden, welche Eindrücke man nach gut zwei Jahren Nutzung und Forschung sammeln konnte und wie der Ausblick für die Zukunft aussieht. Darüber hinaus werden weitere Beispiele für Einrichtungen präsentiert, bei denen alternative Metriken ebenfalls eine praktische Anwendung gefunden haben.

\section{Altmetrics-Forschung}

Seit der Einführung des Begriffs Altmetrics durch Priem et al. (2010) kann die Altmetrics Community auf rund neun Jahre Forschung zu diesem Thema zurückblicken. Einerseits „sind die Sichtbarkeit und Präsenz von Altmetrics durchaus beeindruckend“ (Haustein, 2016a), weil sie vielen Wissenschaftsverlagen als Marketingtool dienen, bereits einige hundert Veröffentlichungen zu diesem Thema existieren, ein eigenes Journal eingeführt wurde und mittlerweile sogar eine eigene Altmetrics-Konferenz abgehal-

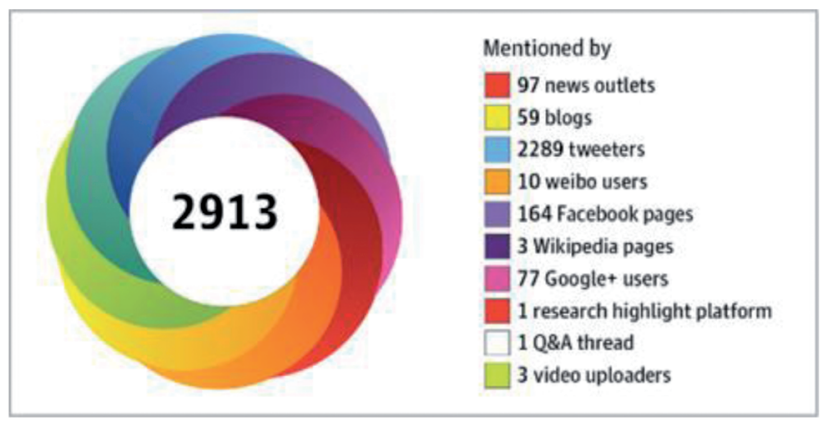

Abbildung 1: Beispiel für die Darstellung des Altmetric-Donut und seine Zusammensetzung.

ten wird. Andererseits fehlt bis dato eine einheitliche Definition und damit auch ein Konsens darüber, was man mit Altmetrics genau misst und welche Aussagen daraus gezogen werden können (Haustein, 2016 b; Franzen, 2017; Butler et al., 2017).

Der Altmetrics Attention-Score wird derzeit in Form des sogenannten „Altmetric-Donut“ von vielen Wissenschaftsverlagen und -einrichtungen als Marketingtool genutzt (s. Nutzung von Altmetrics in Wissenschaftseinrichtungen). Der Donut wurde unter anderem auf den Webseiten der Zeitschriften Nature und Science, sowie in den Repositorien der Universitäten Cambridge und ETH Zürich implementiert. Die Zusammensetzung des Attention-Scores basiert auf einem Algorithmus, der die Aufmerksamkeit von wissenschaftlichem Output in den diversen Quellen unterschiedlich gewichtet addiert ${ }^{1}$.

Dieser Trend wird in der Wissenschaft kritisch betrachtet (Franzen, 2017). Eine einfache Summierung von Counts zu einer einzigen Metrik (composite indicator) ist „problematisch“ (Meier \& Tunger, 2018; European Commission, 2017a). Der Attention-Score bildet nämlich nicht den Impact wissenschaftlicher Leistung ab, sondern dient dazu, die Publikationen herauszufiltern, die ein hohes

1 Siehe hierzu: https://help.altmetric.com/support/solutions/article s/6000060969-how-is-the-altmetric-attention-score-calculated- [7.6. 2019].
*Kontaktperson: Andreas Meier, Forschungszentrum Jülich, WilhelmJohnen-Straße, 52428 Jülich, E-Mail: a.meier@fz-juelich.de

Dr. Dirk Tunger, Forschungszentrum Jülich, Wilhelm-Johnen-Straße, 52428 Jülich, E-Mail: d.tunger@fz-juelich.de 
Maß an Wahrnehmung in den Medien generieren (Warren et al., 2016; European Commission, 2017b).

\section{Machbarkeitsstudie Altmetrics}

Inwiefern und in welcher Weise ist eine Anwendung von Altmetrics in der Wissenschaftspolitik und im Wissenschaftsmanagement sinnvoll? Diese Frage steht im Zentrum einer in Jülich erstellten Machbarkeitsstudie zu Altmetrics (Tunger, Meier \& Hartmann, 2017). Wie werden Altmetrics bereits eingesetzt, $\mathrm{zu}$ welchen Aussagen kommt man und wo zeichnen sich Bottlenecks ab?

Die Machbarkeitsstudie skizziert hierzu vier unterschiedliche Anwendungsfelder und erläutert den jeweiligen Einsatz von Altmetrics:

\section{Wissenschaftsevaluation, Leistungs- bewertung und Messung von Social Impact}

Auf diesem sensiblen Gebiet ist Vorsicht bei der Anwendung von Altmetrics geboten. Die Validität stellt eine essentielle Komponente dar. Im wissenschaftlichen Diskurs müssen ein tiefergehendes Verständnis für die Heterogenität und Aussagekraft der Daten, eine sinnvolle Indikatorik sowie Benchmarks weiter wachsen. Daher werden Altmetrics auch in naher Zukunft eher eine ergänzende Komponente als ein eigenständiger Indikator für die Leistungsbewertung sein. Hinzu kommt, dass einige Forschungsthemen stärker im gesellschaftlichen Fokus stehen als andere, ohne zwingend einen größeren Social Impact aufzuweisen.

\section{Öffentlichkeitsarbeit, Sichtbarkeit und Bewerbung von Aktivitäten}

Ein Teil der Kommunikation über Wissenschaft und deren Wahrnehmung in der Öffentlichkeit wird durch Altmetrics abgebildet. Festzuhalten ist auf jeden Fall, dass Social Media-Aktivität sowohl gemessen an der Häufigkeit der Beiträge als auch der Anzahl von Akteuren in der Tendenz steigt. Damit steigt die Bedeutung und das Potenzial, Social Media-Plattformen heranzuziehen, um proaktiv auf Forschung aufmerksam zu machen bzw. diese zu bewerben.

Institutionelle Bestrebungen beispielsweise von Universitäten oder der Europäischen Kommission sind hier zu beobachten, die gezielt eigene Veröffentlichungen und Aktivitäten ganz im Sinne einer Öffnung des Wissenschafts- systems, eines breiten Wissenstransfers und der Adressierung gesellschaftlicher Herausforderungen platzieren.

\section{Wissenschaft}

Eine Anwendung von Altmetrics in der Wissenschaft kann ähnlich ausgerichtet sein, wie im Abschnitt „Öffentlichkeitsarbeit" beschrieben: Für Wissenschaftler ist die Wahrnehmung ihrer Veröffentlichungen unerlässlich. Das Renommee, das durch die Verwendung ihres wissenschaftlichen Outputs in Form von Ideen, Aussagen, Berechnungen und Ergebnisse durch andere entsteht, ist essentieller Bestandteil des Systems „Wissenschaft“. Erst die Nutzung des Outputs - sei es in anderen wissenschaftlichen Veröffentlichungen oder in webbasierter Kommunikation, Social Media oder Newsbeiträgen und Policy Documents bringt einen nachhaltigen Nutzen für die Forschenden. Es ist zu beachten, dass Wissenschaftlerinnen und Wissenschaftler derzeit noch ohne Social Media erfolgreich arbeiten können, nicht aber ohne wissenschaftliche Veröffentlichungen. Diese sind Teil des wissenschaftlichen Belohnungssystems (s. Diskurs über Altmetrics auf dem Campus). Somit wäre es für Altmetrics aus wissenschaftspolitischer Sicht her förderlich, der Kommunikation und dem Transfer von Wissenschaft in die Gesellschaft einen höheren Stellenwert einzuräumen und entsprechende Anreize für die Wissenschaftlerinnen und Wissenschaftler zu schaffen. Dies würde die Nutzung von Altmetrics fördern und ermöglichen, Fortschritte beim Aspekt des Social Impacts zu erzielen.

\section{Bibliothek}

Mit Altmetrics in Bibliotheken hat sich Kerstin Gimpl (2017) sehr intensiv befasst: In wissenschaftlichen Bibliotheken finden sich häufig die Ansprechpartner einer wissenschaftlichen Einrichtung zu Publikationsdaten und Bibliometrie. Auf sie wird zur Datenbereinigung, zur Zusammenstellung von Publikationsprofilen und zur Datenerhebung im Rahmen von Evaluationen zugegangen. Bibliothekare sind somit Spezialisten für den Umgang mit Daten, vor allem im Zusammenhang mit Publikationen, Nutzerzahlen und das Bestandsmanagement. Altmetrics stellen genau an dieser Stelle ein verbindendes Element dar, weil sie Aufschluss geben über die Nutzung von $\mathrm{Pu}$ blikationen auf den Social Media-Plattformen. Bibliotheken sind also direkt involviert, wenn das Thema „Altmetrics“ an einer Einrichtung aufgegriffen wird (Gimpl, 2017), wie etwa im Forschungszentrum Jülich. 


\section{Nutzung von Altmetrics in Wissenschaftseinrichtungen}

Viele Universitäten und Einrichtungen wie die Universität von Helsinki, die ETH Zürich und die École Polytechnique Fédérale de Lausanne (EPFL) nutzen Altmetrics bereits auf ihren Websites und Repositorien. Ziel ist oft, die Reichweite der eigenen Publikationen auch außerhalb der klassischen Zitationsmetriken zu erkennen. ${ }^{2}$ Das Kommunikationsteam der Duke University nutzt es, um schnell zu erfassen, wie, wo und wann über die Publikationen der Universität in Social Media gesprochen wird. ${ }^{3}$ Die Cambridge University verspricht sich mithilfe von Altmetrics den Impact „im frühen Stadium“ erfassen zu können, da der tatsächliche „klassische“ (bibliometrische) Impact erst in vielen Monaten oder sogar Jahren messbar ist. Auch soll die Aufmerksamkeit, die Projekte, Gruppen oder Departments erhalten, registriert und die „key influencer“ identifiziert werden. Ebenso kann man auf diesem Weg wissenschaftliche Kollaborationen erkennen und den Impact für neuartige Formen der wissenschaftlichen Arbeit (wie Berichte, Software etc.) messen. Das ist mit der klassischen Bibliometrie nicht machbar. ${ }^{4}$

\section{Altmetrics am Forschungszentrum Jülich}

Der Altmetric Explorer von Altmetric.com wurde im Sommer 2017 für drei Jahre lizenziert. Dieser Zeitraum soll als „unverbindliche“ Test- und Erprobungsphase dienen, um Altmetrics besser erforschen und auf dem Jülicher Campus testen zu können. Hierbei ist es allen Jülicher Forschern möglich, den Altmetric Explorer über die IP-Range des Forschungszentrums aufzurufen und zu nutzen. Außerhalb vom Campus kann dies über den VPN-Zugang erfolgen, wie bei allen anderen Datenbanken im Bestand des Forschungszentrums auch. In diesem Rahmen wurde die bereits angesprochene Machbarkeitsstudie erstellt. Doch nicht nur die Nutzung des Altmetric Explorers steht im FZJ im Mittelpunkt. Ebenso interessieren die Einbindung des

2 Siehe hierzu: https://www.altmetric.com/press/press-releases/eur opean-institutions-increasingly-adopting-altmetrics-to-complemen t-existing-bibliometric-analysis/ [7.6.2019].

3 Siehe hierzu: https://www.altmetric.com/case-studies/duke-unive rsity/ [7.6.2019].

4 Siehe hierzu:https://www.research-information.admin.cam.ac.uk/ what-information-available/altmetric-institutions [7.6.2019].
Altmetric Donuts in das Publikationsportal JuSER, die Nutzung von Altmetrics durch die Unternehmenskommunikation (UK) des Forschungszentrums und der Diskurs zwischen den Autoren dieses Beitrags, der UK und den Wissenschaftlern auf dem Campus.

\section{Einbindung von Altmetrics im Publikationsportal JUSER}

Das Publikationsportal JuSER (Juelich Shared Electronic Resources) ist das offizielle Dokumenten-Repositorium des Forschungszentrums Jülich. Es dient als Publikationsdatenbank und beinhaltet Veröffentlichungen Jülicher Wissenschaftlerinnen und Wissenschaftlern. Neben den bibliographischen Metadaten zu der jeweiligen Publikation ist dort auch der sogenannte Altmetric-Badge platziert (s. Abb. 2). Dieser zeigt zum einen den aktuellen Attention-Score der Veröffentlichung, dient aber auch als Link zur detaillierten Beschreibung und Aufschlüsselung im Altmetric Explorer.

\section{Nutzung von Altmetrics durch die Unternehmenskommunikation}

Eine weitere Verwendung finden Altmetrics auch bei der Unternehmenskommunikation des Forschungzentrums: Dort werden besonders interessanten Jülicher Veröffentlichungen eigene Pressemitteilungen gewidmet. Dabei wird auch berücksichtigt, wie die jeweilige Publikation in den Social Media besprochen wird: hierfür wird der Altmetric Badge - ähnlich wie im Publikationsportal JuSER - in die Pressemitteilung integriert. ${ }^{5}$

\section{Diskurs über Altmetrics auf dem Campus}

Bei regelmäßigen Treffen mit der Unternehmenskommunikation sowie den Wissenschaftlerinnen und Wissenschaftlern des Forschungszentrum Jülich werden Altmetrics regelmäßig besprochen und Ideen ausgetauscht ${ }^{6}$. Auch hier wird deutlich, dass der Einsatz und die Akzeptanz der alternativen Metriken sich noch im Anfangsstadi-

5 Siehe als Beispiel hierzu: https://fz-juelich.de/SharedDocs/Presse mitteilungen/UK/DE/2019/2019-02-19-lebensstilgehirn.html;jsessioni $\mathrm{d}=8$ D0403250501D407DC83220231D1DCC8 [7.6.2019].

6 Siehe auch: https://blogs.fz-juelich.de/zweikommazwei/2018/03/ 23/altmetrics-neuer-bibliometriedienst-beleuchtet-zitationen-auf-soc ial-media/ [7.6.2019]. 


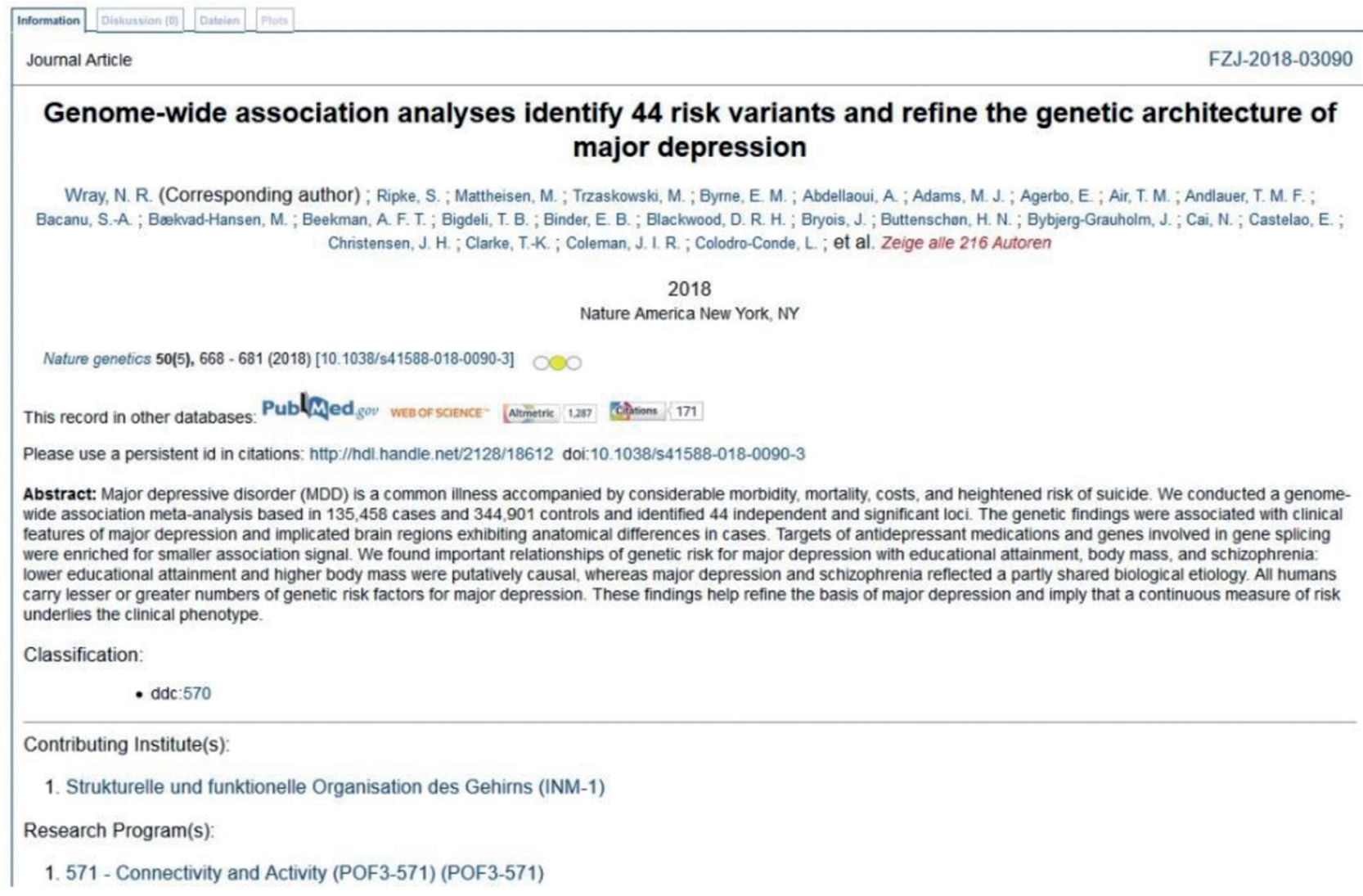

Abbildung 2: Ein Beispiel-Eintrag im Publikationsportal JuSER mit Altmetrics-Badge.

um befinden. Hinzu kommt, dass die Nutzung von Social Media - vor allem bei älteren Wissenschaftlern - nur eine geringe Priorität hat. Als Grund wird genannt, dass die Aktivität in der Wissenschaftskommunikation viel Zeit erfordere und diese neben der wissenschaftlichen Tätigkeit oft fehle. Zudem fehlt vielen Wissenschaftlern der „Anreiz“. Während man anhand von etablierten bibliometrischen Methoden bewertet wird, sind Altmetrics noch keineswegs Teil des Evaluationssystems. Es hat für die meisten Wissenschaftlerinnen und Wissenschaftler schlicht keine Konsequenzen, wenn sie durch Altmetrics nicht gut abgebildet sind. Daher nutzen viele Social Media noch nicht für die Wissenschaftskommunikation. Außerdem scheint es vielen Forschenden schlicht unseriös zu sein, Werbung für die eigene Position oder für eigene Veröffentlichungen in Netzmedien zu machen.

\section{Diskussion und Fazit}

Altmetrics befinden sich noch in der Entwicklung und der Erprobung. Sie sind noch weit davon entfernt, in naher $\mathrm{Zu}$ kunft einen regelmäßigen Beitrag zu quantitativen Wissenschaftsevaluationen zu leisten. Aber: Altmetrics reprä- sentieren Kommunikation, die in der Wissenschaft einen sehr hohen Stellenwert einnimmt und die in zunehmender Weise auch über Fachzeitschriften hinausgeht. Hier anzusetzen und über Anreize nachzudenken, wie neue Formen der Kommunikation gewinnbringend für die Wissenschaft genutzt werden können, ist ein zukunftsweisender Weg. So bleibt beispielsweise zu klären, welche neuen Kommunikationsformen sich in der Wissenschaft durchsetzen und sich neben klassischen Publikationsformen etablieren werden. Dies gilt umso mehr, wenn die Anreize, Wissenschaft mit Hilfe von Social Media in die Gesellschaft zu tragen, erhöht und in das wissenschaftliche Belohnungssystem integriert werden. Wie dargestellt, gibt es auch weitere Einsatzgebiete, in deren Kontext ein Einsatz Sinn macht. Der nächste Schritt kann beispielsweise ein intensiver Dialog mit der Wissenschaft darüber sein, welche neuen Kommunikationsformen sich für die Wissenschaft am besten eignen, welche am besten für einen Dia$\log$ zwischen Wissenschaft und Gesellschaft geeignet sind, beispielsweise beim Transfer von Wissenschaft in die Gesellschaft oder zur Einbindung der Zivilgesellschaft in Wissenschaft. Auch diese Formen von Wissenschaftskommunikation werden in Zukunft ihren Wert und ihren Platz im Wissenschaftssystem haben. 
Digitalisierung wird auch Wissenschaftskommunikation verändern, die Nutzung von Social Media ist ein erster Schritt hierbei. Die Einbindung der Zivilgesellschaft und der Wirtschaft in die Wissenschaft ist ein erklärtes wissenschaftspolitisches Ziel, das sich aus einem neuen Anspruch ergibt, wie wir gemeinsam unsere Zukunft gestalten: „Nur gemeinsam machen wir die Digitalisierung zum Erfolg. Deshalb setzen wir auf Partnerschaften und Dialog mit und zwischen der Wissenschaft, der Wirtschaft und der Gesellschaft in Deutschland, Europa und darüber hinaus" (Digitalstrategie des $\mathrm{BMBF}^{7}$, Seite 5). Aufmerksamkeit für Wissenschaftlerinnen und Wissenschaftler wird sich in Zukunft nicht nur auf klassische wissenschaftliche Publikationen beschränken, sondern auch neue Formen von Kommunikation (z. B. Netzquellen, Blogs, Newsbeiträge, Policy Documents etc.) und Sharing Economy (z.B. freier Zugang zu wissenschaftlichen Publikationen oder Forschungsdaten) beinhalten. Aufmerksamkeit insgesamt wird für Wissenschaft einen hohen Stellenwert, wenn nicht sogar einen höheren als aktuell, haben ${ }^{8}$.

Deskriptoren: Altmetrics, Bibliometrie, Social Media, Wissenschaft, Kommunikation

\section{Literatur}

Butler, J.S., Kaye, I. D., Sebastian, A.S., Wagner, S. C., Morrissey, P. B., Schroeder, G. D., Kepler, C. K. \& Vaccaro, A. R. 2017. The Evolution of Current Research Impact Metrics: From Bibliometrics to Altmetrics? Clinical Spine Surgery, 30(5).

European Commission 2017a. Next-generation metrics: Responsible metrics and evaluation for open science. doi:10.2777/337729

European Commission 2017b. Mutual Learning Exercise: Open Science Altmetrics and Rewards.

Franzen, M. 2017. Digitale Resonanz. Neue Bewertungskulturen fordern die Wissenschaft heraus. WZB Mitteilungen 155 , S. 30-33.

Gimpl, K., 2017. Evaluation von ausgewählten Altmetrics-Diensten für den Einsatz an wissenschaftlichen Bibliotheken. Master-Thesis im Studiengang Bibliotheks- und Informationswissenschaft / MALIS (Master in Library and Information Science); Technische Hochschule Köln, Fakultät für Informations- und Kommunikationswissenschaften, urn:nbn:de:hbz:79pbc-opus-10341

Haustein, S. 2016a. Vier Tage für fünf Jahre Altmetrics. Bericht über die Konferenz 2AM und den Workshop altmetrics15. b.i.t. online, 19(1): 110-112.
Haustein, S. 2016b. Grand challenges in altmetrics: heterogeneity, data quality and dependencies. Scientometrics. doi: 10.1007/ s11192-016-1910-9

Meier, A., \& Tunger, D. 2018. Investigating the transparency and influenceability of altmetrics using the example of the RG score and the ResearchGate platform. Information Services and Use, 38(1-2), 99-110. https://doi.org/10.3233/ISU-180001

Priem, J., Taraborelli, D., Groth, P., \& Neylon, C. 2010. alt-metrics: $a$ manifesto. Retrieved August 15, 2011, from http://altmetrics.or $\mathrm{g} /$ manifesto/

Tunger, D.; Meier, A.; Hartmann, D. 2017.Machbarkeitsstudie Altmetrics. http://hdl.handle.net/2128/16419

Warren, H.R.; Raison, N.; Dasgupta, P. 2016. The Rise of Altmetrics. Journal of the American Medical Association, 317(2), S. 131-132.

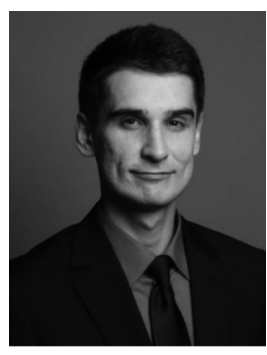

\section{Andreas Meier}

Forschungszentrum Jülich

Wilhelm-Johnen-Straße

52428 Jülich

a.meier@fz-juelich.de

Andreas Meier studierte Informationswissenschaft und Sprachtechnologie an der Heinrich Heine Universität Düsseldorf. Seit 2013 ist er Mitglied des Bibliometrie-Teams an der Zentralbibliothek des Forschungszentrums Jülich. Neben der Bibliometrie befasst er sich u. a. mit dem Thema Altmetrics, das auch im Mittelpunkt seiner Masterarbeit steht.

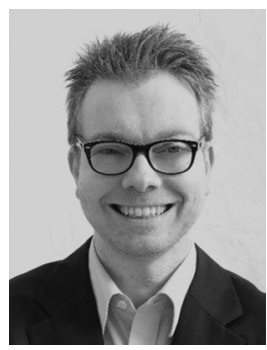

\section{Dr. Dirk Tunger}

Forschungszentrum Jülich

Wilhelm-Johnen-Straße

52428 Jülich

d.tunger@fz-juelich.de

Dr. Dirk Tunger ist promovierter Informationswissenschaftler und war bis 2017 mit dem Aufbau und der Leitung des Teams „Bibliometrie“ in der Zentralbibliothek des Forschungszentrums Jülich betraut. Seit 2018 ist er wissenschaftlicher Mitarbeiter im Kompetenzzentrum Analysen, Studien, Strategien des Projektträgers im Forschungszentrum Jülich. Seine thematischen Schwerpunkte sind vor allem Foresight, Trend- und Wirkungsanalyse.
7 https://www.bildung-forschung.digital/de/die-digitalstrategiedes-bmbf-2479.html [26.6.2019].

8 Siehe auch: https://www.wissenschaftskommunikation.de/altmet rics-kommt-die-oekonomie-der-aufmerksamkeit-21609/ [7.6.2019]. 